\title{
Microgrids Emergency Management Exploiting EV, Demand Response and Energy Storage Units
}

\author{
C. Gouveia, C. L. Moreira, J. A. Peças Lopes, Senior Member, IEEE \\ INESC TEC - INESC Technology and Science (formerly INESC Porto) \\ Porto, Portugal. \\ cstg@inescporto.pt, cmoreira@inescporto.pt,jpl@fe.up.pt
}

\begin{abstract}
Within the Smart Grid paradigm, the MicroGrid concept (MG) presents an adequate framework to monitor and manage the low voltage network and coordinate the resources connected to it, including the smart grid new players, namely the consumers, prosumers and the Electric Vehicles (EV). The coordinated management and control of the MG resources, enables the operation both connected to the main power network or autonomously, due to planned or unplanned outages. In order to operate autonomously, the MG relies in its storage capacity to provide some form of energy buffering capabilities to balance load and generation. This paper presents innovative methodology to coordinate the microgrid storage capacity with EV smart charging strategies and demand response schemes, in order to improve microgrid resilience in the moments subsequent to islanding and reduce the non-served load. The effectiveness of the proposed algorithms are validated though extensive numerical simulations.
\end{abstract}

Index Terms-- Demand Response, Electrical Vehicles, Energy Storage, Islanding Operation, MicroGrid, Vehicle-to-Grid.

\section{I.INTRODUCTION}

Microgrids (MG) are Low Voltage (LV) distribution systems, integrating distributed energy sources, storage devices, controllable loads and Electric Vehicles (EV) [1], [2]. As shown in Fig.1, the MG architecture is composed of a network of controllers with local intelligence headed by the MG central controller (MGCC), which is responsible for monitoring and coordinating all the MG resources. In the local control layer, there are three types of controllers: the load controller (LC), the Microsource (MS) controller (MC) and the EV controller (VC) [2], [3].

Being an extremely flexible cell of the electric power system, the MG is able to operate interconnected to the main power system or autonomously in case of emergency situations, if endowed with proper control and management system. Under such conditions, the MG relies in the fast response of the local controllers for microgeneration dispatch, storage devices energy management and for the activation of load shedding mechanisms [2].

This work is financed by the ERDF- European Regional Developmen Fund through the COMPETE Program (operational program for competitiveness) and by National Funds through the FCT - Fundação para a Ciência e a Tecnologia (Portuguese Foundation for Science and Technology) within Project Microgrids+EV: Identification of Control and Management Strategies for Microgrids with Plugged-in Electric Vehicles -ref. PTDC/EEA-EEL/103546/2008-(FCOMP-01-0124-FEDER-009866).

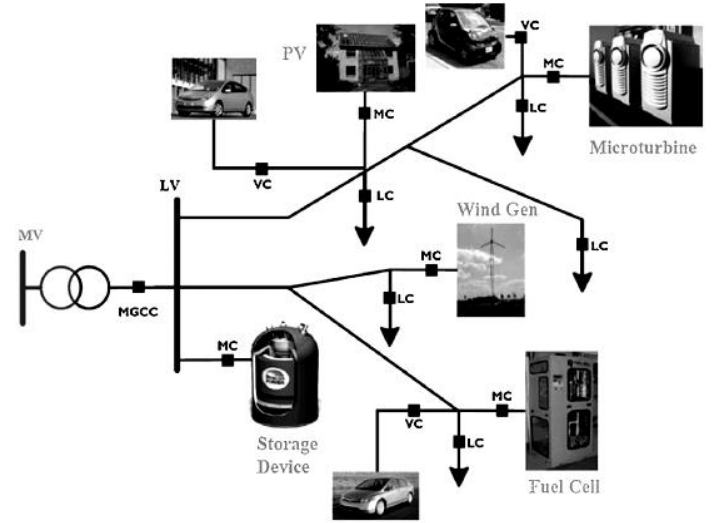

Figure 1. Basic architecture of a microgrid [3].

Ensuring a successful MG autonomous operation is quite challenging. The success of the MG islanding will depend on the system pre-fault operating conditions, namely: on the MG storage capacity, microgeneration reserve capacity and on the flexibility of the MG loads. The deployment of EV connected to the LV network and the implementation of smart charging strategies has been shown to reduce the central storage capacity, required to assure MG system robustness during islanding conditions [3], [4]. However, the participation of EV will depend on the number of EV connected to the system.

In addition to the control flexibility provided by $\mathrm{EV}$, the development of innovative emergency demand response schemes for MG islanded operation might improve the security of operation. Exploring direct load control strategies for the provision of primary and secondary frequency reserves has been proposed in [5]-[8]. The results have shown that frequency responsive load strategies improve the overall power system stability, when facing power disturbances. Therefore, the implementation of emergency demand response schemes coordinated with the participation of EV and MG generation resources will allow a more efficient and secure management of the MG under emergency conditions.

Taking into consideration this general idea, this paper presents a new framework for the MG management and 
control considering the integration of EV and emergency demand response schemes. An innovative approach is proposed to define the most adequate strategies for managing the $\mathrm{MG}$ resources during islanding operation.

\section{II.ANALYZING THE MG CAPACITY TO ENSURE SUCCESSFUL ISLANDING}

When the MG disconnects from the upstream MV grid, it is necessary to establish the voltage reference in magnitude and frequency. In order to maintain power balance between generation and load and restore frequency to adequate values, specific primary and secondary frequency control strategies are required [2].

The MG primary frequency response is ensured by the MG storage unit, such as flywheels or ultracapacitors, which have the capability of providing fast power balance (ie. flywheels, ultracapacitors, etc.). The storage unit coupling inverter is controlled as a voltage source inverter with external droop control loops, defining the MG voltage and frequency references as a function of the grid operating conditions, as in (1),

$$
\begin{aligned}
& \omega=\omega_{0}-k_{P} \times P \\
& V=V_{0}-k_{Q} \times Q
\end{aligned}
$$

where $P$ and $Q$ are the inverter active and reactive power outputs, $k_{P}$ and $k_{Q}$ are the droop slopes (positive quantities) and $\omega_{0}$ and $V_{0}$ are the idle values of the angular frequency and voltage (values of the inverter angular frequency and terminal voltage at no load conditions).

In order to correct the MG frequency deviation, secondary frequency control is required. The MG secondary frequency control is ensured by the controllable microgeneration (i.e. microturbines or fuel cells), which will change their power output in order to compensate the power injected/absorbed by the storage unit(s). The MG secondary frequency control can be implemented locally or centralized at the MGCC. If a centralized secondary frequency control is adopted, the microgeneration dispatch can be defined according to the reserve capacity of the controllable unit, as shown in (2)

$$
\left\{\begin{array}{l}
d P_{i}=P_{\text {unbalance }} \frac{R_{i}}{R} \\
\sum_{i=1}^{n} d P i=P_{\text {unbalance }}
\end{array}\right.
$$

where $d P_{i}$ is the emergency active power step of unit $i$, $P_{\text {unbalance }}$ is the active power unbalance after islanding, $R_{i}$ is the reserve capacity of unit $i$ and $R$ is the MG total reserve capacity.

The MG secondary control will only recover the MG frequency to nominal values if the microgeneration reserve capacity is sufficient to supply the MG loads. Otherwise, it will be necessary to shed a portion of the MG load. The MG load shedding schemes are usually implemented in steps which are activated based on under-frequency or rate-ofchange-frequency relays [2]. In [8], a central demand response strategy for the MG primary frequency regulation was proposed. The algorithm developed determines the minimum percentage of electric water heaters which should be disconnected/connected. Based on the MG frequency behavior following a disturbance, the algorithm activates one of two control modes: mode 1 is activated when the frequency deviation surpasses an admissible range, sending a disconnection/reconnection command to the electric water heaters; mode 2 is activated when the frequency returns to the admissible range sending reconnection/disconnection orders to the loads participating in mode. This method requires a robust communication network between the central controller and the load controllers, in order to ensure the load control within acceptable time. Load control methods based on local frequency measurements [5]-[7] avoid the dependence from communication infrastructures, requiring only an accurate local frequency measurement.

Regarding the control of EV, in [3], [4] the authors propose an active power/frequency droop control strategy implemented at the EV coupling inverter, where the EV will change the power exchanged with the LV grid based on the MG frequency, as shown in Fig.2. When the generation exceeds the load, the frequency will rise. In order to compensate the power unbalance, the EV will increase its charging power from the reference power $\left(P_{r e f}\right)$ up to the maximum charging power $\left(P_{\max }\right)$. If the $\mathrm{MG}$ load exceeds generation the $\mathrm{MG}$ frequency will decrease and the $\mathrm{EV}$ reduces its power consumption, reducing the load of the system. For large disturbances, causing the frequency to go below the zero-crossing frequency $\left(f_{0}\right)$, the EV starts to inject power into the grid (Vehicle-to-Grid - V2G - functionality).

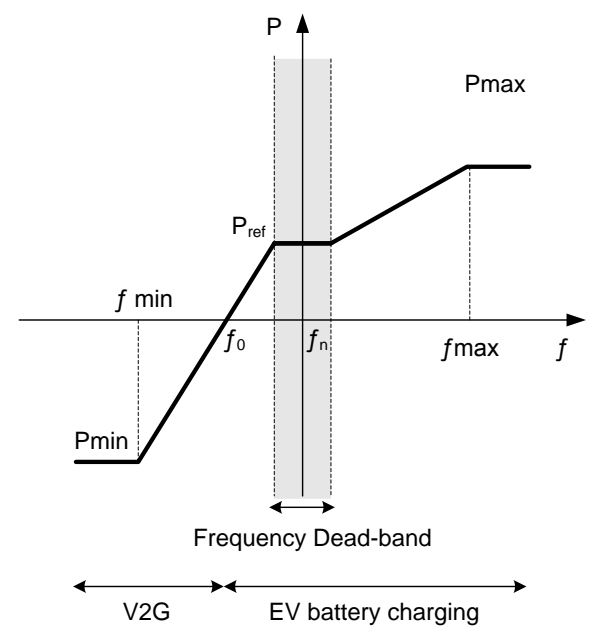

Figure 2. EV Active Power-frequency droop characteristic [3], [4].

Considering the MG frequency regulation strategies presented in this section, it is possible to conclude that the effectiveness of the MG emergency control strategies, in the moments subsequent to the islanding, will depend on the prefault operating conditions, characterized by the following variables: 
- MG storage capacity, which is essential to ensure primary frequency regulation.

- Controllable MS reserve capacity, in order to perform secondary frequency regulation, allowing the frequency to return to nominal values.

- Non-controllable MS power production, which acts as a negative load in the system.

- $\quad$ MG flexible load including the EV connected to the LV network, which can improve the MG frequency, either by shedding interruptible loads or through autonomous operation of $\mathrm{EV}$.

- MG load conditions in comparison to total generation, thus defining the system imbalance prior to islanding.

In order to ensure the success of the MG emergency control strategies, these variables have to be monitored and coordinated through adequate functionalities. The algorithm presented in next section analyzes the MG operating conditions based on the data periodically sent by the local controllers and then identifies the most appropriate actions to ensure a secure islanding.

\section{III.MG EMERGENCY MANAGEMENT TOOL}

The algorithm proposed in this paper consists in four main steps, namely:

1) Characterize the MG operating state based on the information sent by local controllers. The algorithm determines the MG storage capacity, microgeneration reserve capacity, power flow between the $\mathrm{MG}$ and the MV network and load (divided between the controllable and non-controllable load).

2) Determine the severity of the disturbance, which is characterized by the active power unbalance resulting from the difference between the power imported from the main grid $\left(P_{\text {unbalance }}\right)$ and the total reserve capacity from controllable MS $(R)$.

3) Determine the amount of load to shed. In case the MG does not have enough reserve capacity, it is necessary to exploit emergency responsive loads and shed some part of the MG load in order to ensure power balance.

4) Evaluate the security of the MG during an unplanned islanding. The algorithm determines if the MG has enough storage capacity to ensure power balance during the time required for restoring the frequency to nominal values or if the frequency goes out of admissible limits. Otherwise, it might be necessary to shed some load temporarily.

In step 3) the amount of load to shed $\left(\Delta P_{\text {shed }}\right)$ is determined based on the MG reserve capacity (R) and on the active power unbalance after islanding $\left(P_{\text {unbalance }}\right)$, as in (3).

$$
\Delta P_{\text {shed }}=R-P_{\text {unbalance }}
$$

During islanding conditions, if the MG lacks energy to ensure power balance, the system will collapse. The energy balance in the storage unit $(E)$ can be determined by (4),

$$
E=E_{\Delta P}-E_{M S}-E_{V E}-E_{D R}
$$

where, $E_{\Delta P}$ is the energy required to compensate the power unbalance resultant from the $\mathrm{MG}$ disturbance, $E_{\mathrm{MS}}$ is the energy provided by the microgeneration units, $E_{V E}$ is the energy resultant from the response of the EV, and $E_{D R}$ is the contribution of the temporary load curtailment if required.

The energy provided by the microgeneration units will depend on the action of the secondary frequency control. In this case it was considered the centralized method described in section II. The power response of the MG controllable microgeneration units to the secondary control dispatch is non-linear, requiring the adoption of the microsources dynamic model, as in [9]. Since the storage energy $(E)$ will also depend on the microgeneration response, the $\mathrm{MG}$ simplified dynamic model presented in Fig.3 was developed. The model adopted ignores the LV network and the dynamics of the power converters, which are faster than the MG dynamic behavior.

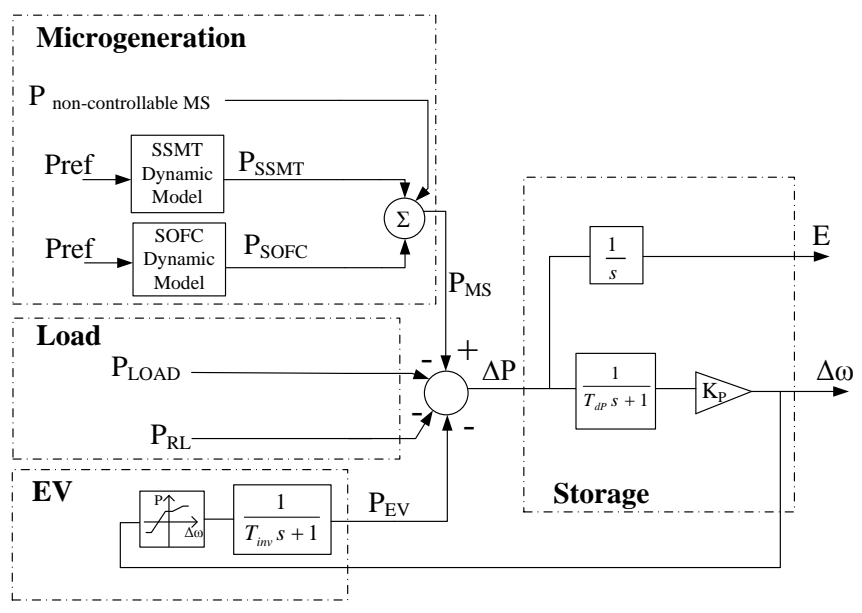

Figure 3. MG simplified dynamic model.

After step 4), if the algorithm identifies that there isn't enough storage capacity or the frequency goes out of admissible limits it might be necessary to consider an additional load curtailment. However, contrarily to step 3), the load could be reconnected after the frequency returns to admissible values. It is expected that this temporary load curtailment will improve the $\mathrm{MG}$ frequency regulation capacity and reduce the solicitation from the storage unit(s).

\section{A. EV and Load control strategy}

When operating islanding, the MG can take advantage of its load resources flexibility to improve its frequency regulation capacity. In order to minimize the consumer's discomfort, this paper proposes a selective load control scheme, considering the EV connected to the system and availability of controllable loads.

The EV connected to the system can be distinguished according to the controllability of its charging mode. Conventional EV chargers, such as the ones available in the market, do not allow the implementation of active powerfrequency droop presented in Fig.2. In this case, the EV behaves has a conventional (constant) load, allowing only the 
implementation of on/off control, usually referred as dumb charging mode. However, the deployment of bi-directional smart chargers will enable the implementation of active power-frequency droop characteristics, where the EV will autonomously respond to the MG frequency deviations.

The MGCC will use the information sent by the EV controllers (VC), differentiating between dumb charging and smart charging EV adherents. Dumb charging EV with SOC higher than $80 \%$ will be considered candidates for load shedding. While in the case of smart charging EV, the MGCC is able to change the frequency and power parameters of the droop characteristics, without requiring their disconnection.

Regarding the MG loads, the LC should be equipped with a dedicated functionality to manage controllable loads. Through a user interface, the consumer may prioritize the availability of its home appliances to participate in the MG load control scheme. The on/off control of loads is based on the LC frequency measurement. In order to avoid overshedding, the algorithm defines the disconnection frequency to the selected loads, based on the frequency deviation estimated through the simplified dynamic model.

At the MGCC level this information will be processed and aggregated, as shown in Fig.4. The loads are grouped according to the consumer's preferences. The MG emergency load control strategy will start by selecting the loads which consumers selected as less priority loads.

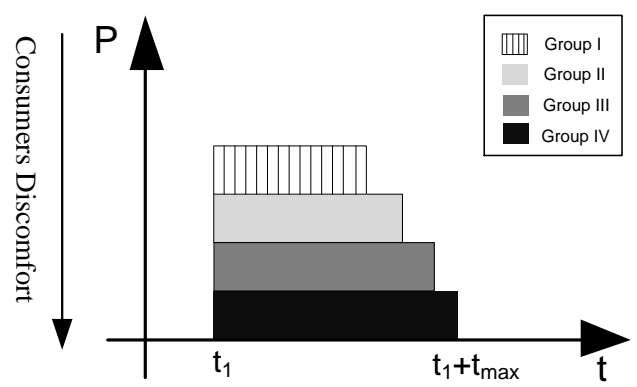

Figure 4. General structure of the MGCC aggregated load control availability.

\section{IV.MICROGRID TEST SYSTEM}

The MG emergency control strategy presented in this paper was tested for a typical urban LV distribution network represented in Fig.5. The LV network has a nominal voltage of $400 \mathrm{~V}$ and supplies a total of 92 clients, from which 69 have a single-phase connection and the remaining 24 have a three-phase connection to the LV network. A 3.6 MJ flywheel capable of injecting $100 \mathrm{~kW}$ maximum power was placed at the MV/LV substation. The MG has four controllable microgeneration units, namely: one Solid Oxide Fuel Cell (SOFC) and three Single-Shaft Microturbines (SSMT). Renewable based microgeneration units such as photovoltaic panels and micro-wind turbines were also considered. The MG generation and load conditions are summarized in Table I.

The EV are assumed to be connected to the MG through single-phase chargers, taking about 8-12 $\mathrm{h}$ to reach full autonomy, offering higher control flexibility. At the nominal frequency the power absorbed was considered $75 \%$ of the nominal power (about $3 \mathrm{~kW}$ ). The $\mathrm{EV}$ frequency-droop parameters are presented in Table II. The aggregated load control availability considered for the load control strategy is defined in Table III.

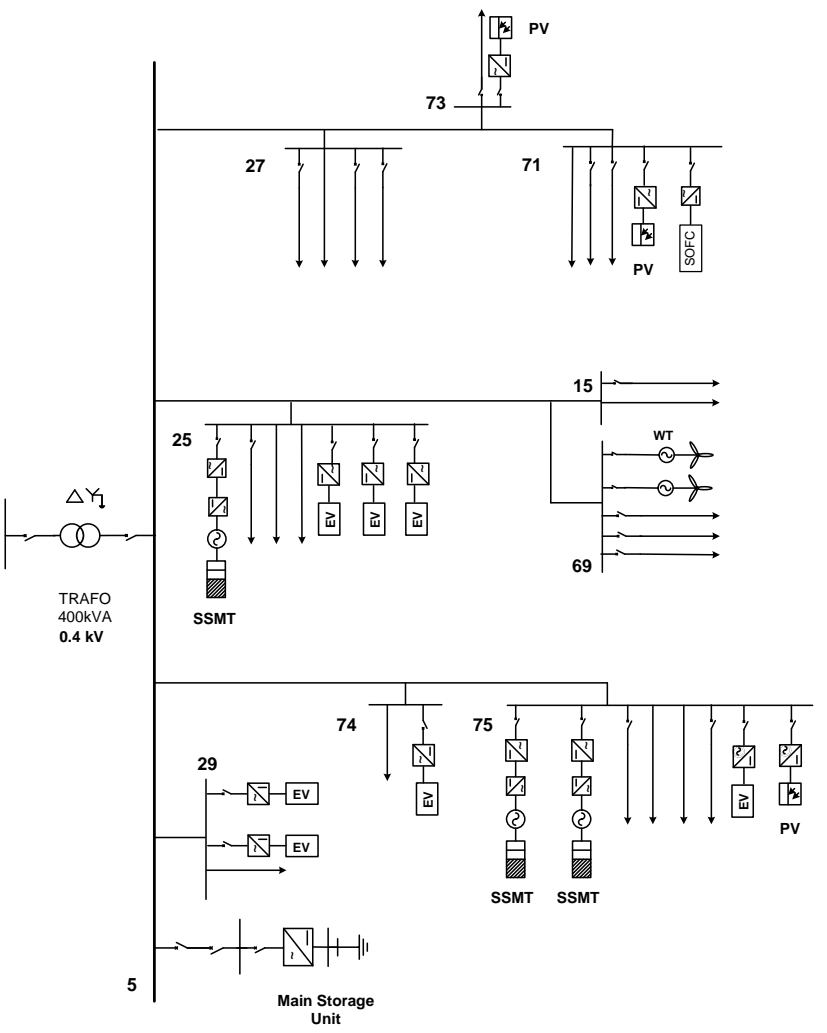

Figure 5. MG test system.

Table I. MG GENERATION CAPACITY AND LOAD Type of Connection

\begin{tabular}{c|ccccc}
\cline { 2 - 6 } \multicolumn{2}{c}{} & \multicolumn{5}{c}{ Type of Connection } \\
\hline Load (kW) & 42 & 20 & 25 & 33 & 120 \\
MS (kW) & 120 & 8 & 16 & 12 & 156 \\
E.V. (kW) & - & 15 & 9 & 12 & 36 \\
\hline
\end{tabular}

Table II. EV FREQUENCY- DROOP PARAMETERS

\begin{tabular}{lc}
\hline Frequency-Droop Parameters & Values \\
\hline Nominal Frequency $(\mathrm{Hz})$ & 50 \\
Zero-Crossing Frequency $(\mathrm{Hz})$ & 49.5 \\
Maximum Frequency $(\mathrm{Hz})$ & 51 \\
Minimum Frequency $(\mathrm{Hz})$ & 49 \\
Frequency Dead-band $(\mathrm{Hz})$ & 0.2 \\
\hline
\end{tabular}

TABLE III. CONTROLLABLE LOAD AVAILABILITY

\begin{tabular}{cc}
\hline Load Group & $\mathrm{P}(\mathrm{kW})$ \\
\hline I & 30,2 \\
II & 14,3 \\
III & 18,3 \\
\hline
\end{tabular}

\section{V.SIMULATION RESULTS AND DISCUSSION}

In order to demonstrate the potential of the emergency control of responsive loads, two scenarios were considered. The first scenario considers that the MG lacks sufficient reserve capacity to supply the MG load after the islanding, 
while in the second scenario the MG has sufficient reserve capacity to supply the MG loads. In both scenarios the MG was considered to be importing power from the MV network.

\section{A. Scenario 1-MG with reserve capacity shortage}

In this scenario the SOFC is disconnected from the MG and the SSMT are producing approximately half of their nominal power. Consequently, the MG is importing $77.2 \mathrm{~kW}$ of active power. All the EV are controlled with active power/frequency droop.

Since the amount of power imported from the main grid is higher than the MG reserve capacity, the algorithm proposed in section III defines a $36.3 \mathrm{~kW}$ of total load shedding, corresponding to the loads of group I and $42.3 \%$ of the loads from group II. These loads will be disconnected when the frequency reaches $49.7 \mathrm{~Hz}$ and will only be reconnected when the MG resynchronizes with the MV network. The frequency set-point was defined to a value higher than the minimum frequency estimated by the energy balance model in order to avoid a larger frequency disturbance.

Fig.6 compares the MG frequency with and without the load control strategy. If the load is not curtailed the MG frequency will stabilize at $49.75 \mathrm{~Hz}$. Therefore, as shown in Fig.7 the storage unit will continue to inject of power corresponding to the unbalance between load and generation. Due to the limited storage capacity, the MG would collapse after100s.

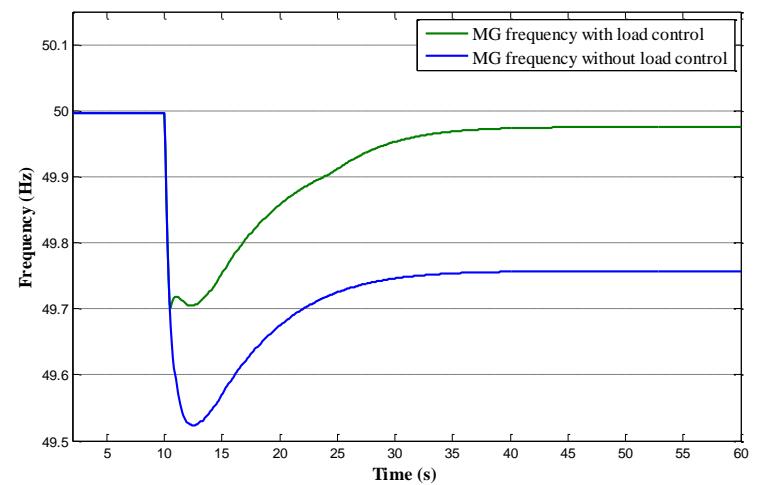

Figure 6. MG frequency after the islanding with no load control (blue) and with the defined load shedding strategy (green).

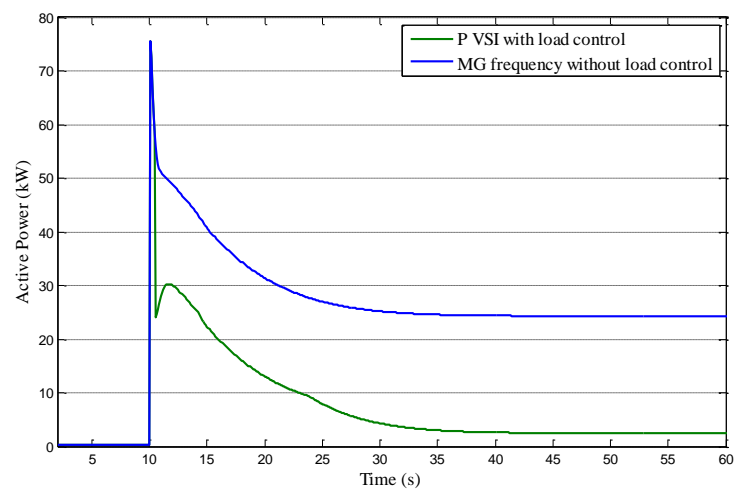

Figure 7. Storage unit power response after the islanding with no load control (blue) and with the defined load shedding strategy (green).

\section{B. Scenario 2-MG with sufficient reserve capacity to supply the $M G$ loads}

In this scenario the SOFC in node 71 is connected to the MG producing approximately half of their nominal power. In normal interconnected mode the MG is importing less power when compared to the first scenario (approximately $52 \mathrm{~kW}$ of active power). If an unplanned islanding occurs, the MG has a total MS reserve capacity of $55.4 \mathrm{~kW}$, not requiring the curtailment of load during the MG islanding. However, the effect of temporary load curtailment is investigated.

Fig. 8 compares the MG frequency response considering different scenarios of EV controllability, namely: a case with $100 \%$ of the EV controlled through P-f droop, a second case where $50 \%$ of the EV adopt a dumb charging mode and a third case where all the EV connected to the MG adopt a dumb charging mode. As it was expected, the worst case is obtained when the EV do not participate in the MG primary frequency regulation. In this case the frequency reaches a minimum of $49.46 \mathrm{~Hz}$. The participation of EV decreases the initial frequency deviation to $49.66 \mathrm{~Hz}$, consequently decreasing the power required to be injected by the storage unit.

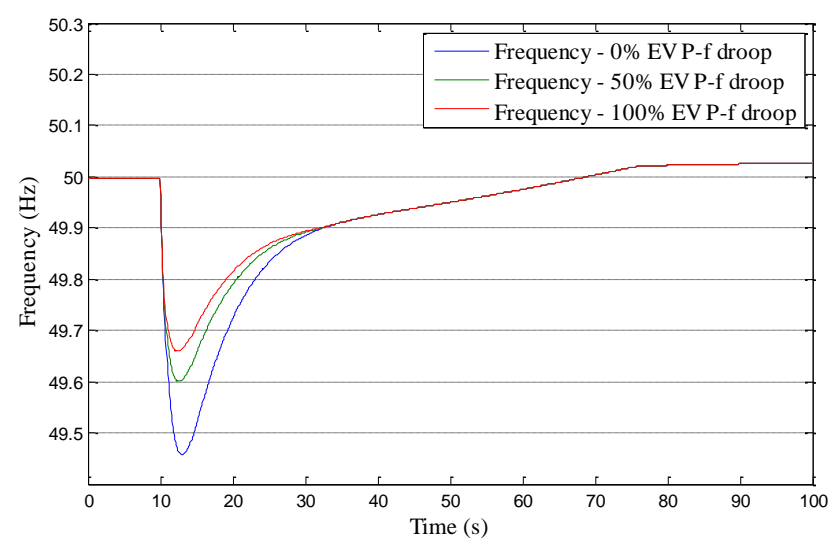

Figure 8. MG frequency after the islanding with $0 \%$ of the (blue), $50 \%$ (green) and $100 \%$ (red) of EV participating in the MG primary frequency regulation.

Fig.9 shows the MG frequency when considering the temporary curtailment of the loads from Group I (Table III), considering the different scenarios of EV charging modes. Since the MG has enough MS reserve capacity to supply the MG loads, the loads will be reconnected when the frequency recovers to values higher than $49.8 \mathrm{~Hz}$. A random delay was applied to the loads in order to avoid severe frequency transients.

As shown in Fig.9 and Fig.10, the participation of the EV and load in the MG primary frequency regulation reduces the severity of the frequency disturbance, consequently decreasing the storage solicitation. Further improvements on the reconnection of loads can be performed in order to minimize the transients. However, the MG frequency response will also depend in the moment of loads' reconnection. 


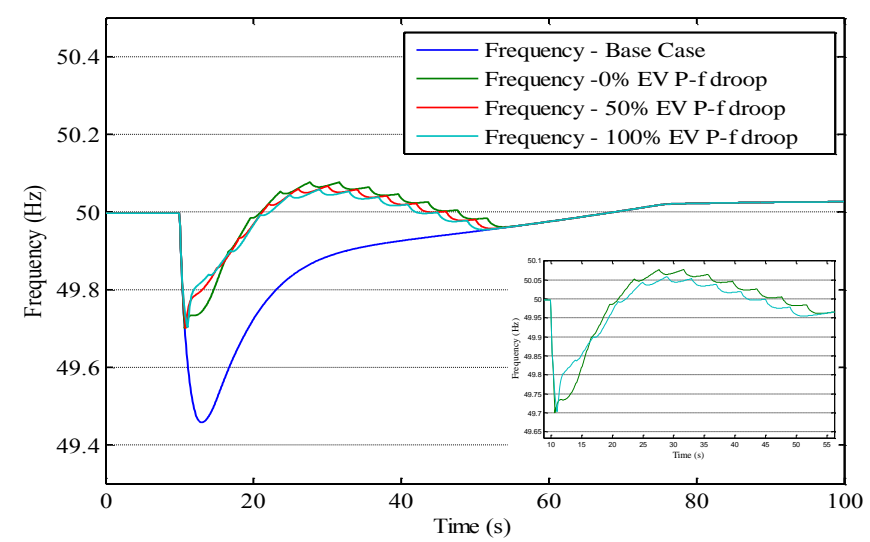

Figure 9. MG frequency after the islanding with the emergency load control strategy and participation of EV.

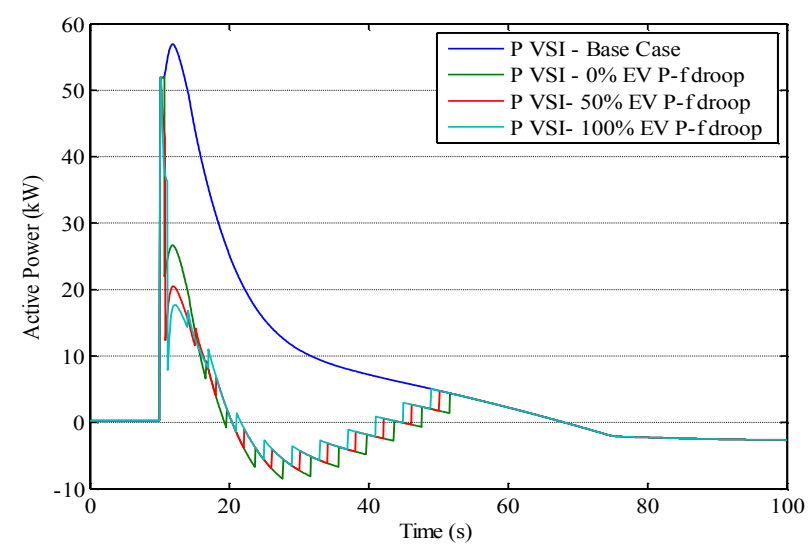

Figure 10. Storage unit active power injection after the islanding with the emergency load control strategy and participation of EV.

\section{Influence of the MG storage unit State Of Charge}

The severity of the power disturbance during the islanding will depend on the MG pre-fault conditions. If the MS power output is low, the MG will need to import more power from the main grid, consequently leading to a higher power unbalance. Fig. 11 shows the response of the storage unit when the MG was importing $100 \mathrm{~kW}$ from the MV network. In this case we have considered the same conditions of scenario 2, changing only the initial power output of the SSMT and SOFC units.

The most severe case is when neither the EV nor the emergency demand response is considered (blue line), requiring a total energy of approximately $3 \mathrm{MJ}$. This means that if the storage unit state of charge was inferior to $85 \%$ the storage would run out of energy. However, if all the EV connected to the MG participates in the primary frequency regulation (red), the total energy required will be approximately $2.26 \mathrm{MJ}$, which means that we only need to curtail more load when the storage unit has a SOC inferior to $65 \%$. Finally, the participation of EV in coordination with the emergency demand response scheme will reduce the energy required to ensure power balance to $40 \%$ of the storage unit total capacity.

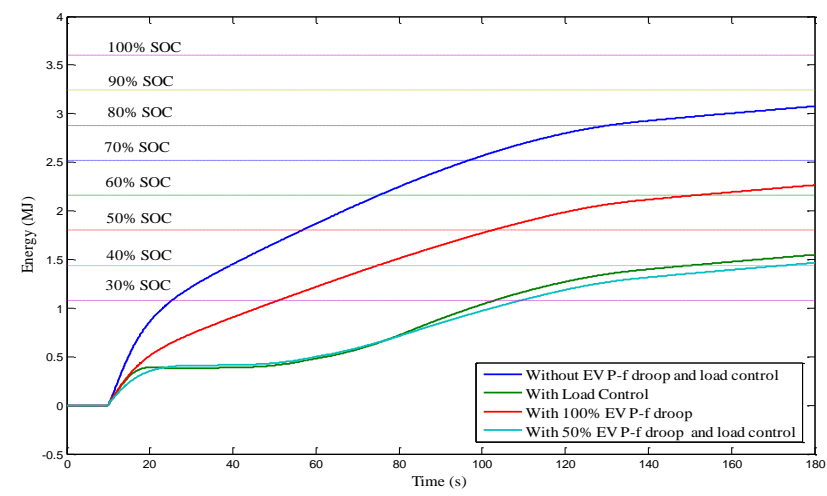

Figure 11. Energy supplied by the storage unit for scenario 2 with a 100kW power import from the MV network.

\section{VI.CONCLUSIONS}

This paper presents the development of a MG power management tool, which coordinates the microgrid storage management with EV smart charging strategies and demand response schemes. The tool runs online at the MGCC, periodically updating the EV and load control strategies according to the MG state, taking into account the severity of the islanding disturbance and the limited energy storage capacity. From the results obtained in a LV test MG it is possible to conclude that the derived approach improved the MG primary frequency regulation capacity increasing the robustness of the MG control architecture. The participation of the MG loads based on local frequency measurements has proven to be effective in the provision of primary reserve. However, further improvements on the local implementation of this load control scheme is required.

\section{REFERENCES}

[1] R.H. Lasseter, "Smart Distribution: Coupled Microgrids," Proceedings of the IEEE, vol.99, no.6, pp.1074-1082, June 2011.

[2] J.A.P. Lopes, C.L. Moreira, AG. Madureira, "Defining control strategies for MicroGrids islanded operation". IEEE Transactions on Power Systems, vol.21, no.2, pp. 916- 924, May 2006.

[3] J.A. Pecas Lopes, Silvan A. Polenz, C.L. Moreira, Rachid Cherkaoui, "Identification of control and management strategies for LV unbalanced microgrids with plugged-in electric vehicles", Electric Power Systems Research, vol. 80, Issue 8, pp. 898-906, August 2010,

[4] J.A.P. Lopes, F.J. Soares, P.M.R. Almeida, "Integration of Electric Vehicles in the Electric Power System," Proceedings of the IEEE, vol.99, no.1, pp.168-183, Jan. 2011

[5] F.C. Schweppe, R.D. Tabors, J.L. Kirtley, H.R.Outhred, F.H. Pickel, A.J. Cox, "Homeostatic Utility Control," IEEE Transactions on Power Apparatus and Systems, vol.PAS-99, no.3, pp.1151-1163, May 1980.

[6] K. Samarakoon, J. Ekanayake, N. Jenkins, "Investigation of Domestic Load Control to Provide Primary Frequency Response Using Smart Meters," IEEE Transactions on Smart Grid, vol.3, no.1, pp.282-292, March 2012.

[7] J.A. Short, D.G. Infield, L.L. Freris,"Stabilization of Grid Frequency Through Dynamic Demand Control," IEEE Transactions on Power Systems, vol.22, no.3, pp.1284-1293, Aug. 2007.

[8] S.A. Pourmousavi, M.H. Nehrir, "Real-Time Central Demand Response for Primary Frequency Regulation in Microgrids", IEEE Transactions on Smart Grid, vol.3, no.4, pp.1988-1996, Dec. 2012.

[9] A. Keyhani and M. Marwali,"Microgrids Operation and Control under Emergency Conditions", in Smart Power Grids 2011, Ed. Berlin, Springer-Verlag, 2012, ch. 12, pp. 351-399. 
(C) 2013 IEEE. Personal use of this material is permitted. Permission from IEEE must be obtained for all other uses, in any current or future media, including reprinting/republishing this material for advertising or promotional purposes, creating new collective works, for resale or redistribution to servers or lists, or reuse of any copyrighted component of this work in other works. 